\title{
Evaluación de una prueba para valorar el razonamiento clínico en diferentes niveles de capacitación médica
}

\author{
GABRIEL BAÑO ${ }^{1}$, SANDRA DI LALLA ${ }^{1}$, PAULA DOMÍNGUEZ ${ }^{1}$, \\ MARÍA NOEL SEOANE ${ }^{1}$, RAQUEL WAINSZTEIN ${ }^{2}$, \\ MARÍA FABIANA OSSORIO ${ }^{1,2}$, FERNANDO FERRERO ${ }^{1,2}$
}

\section{Assessing a test to evaluate clinical analytical thinking according to medical training level}

\begin{abstract}
Background: MATCH (Measuring Analytical Thinking in Clinical Health Care) is an instrument to evaluate clinical reasoning. Aim: To assess MATCH performance in professionals and students with different training in pediatrics. Material and Methods: MATCH was administered to medical students (S), first (R1) and third $(R 3)$ year residents and staff physicians $(P)$. We evaluated the score and time required to achieve it, according to training level in pediatrics. Results: Eighty five subjects were included ( $23 \mathrm{~S}, 28 \mathrm{R} 1,17 \mathrm{R} 3$ y $17 \mathrm{P}$ ), achieving $37.4 \pm 6.0$ points, in $25.2 \pm 8.5$ minutes. There were significant differences in score and time, according to training level. There was a positive correlation between training level and score (Rho $=0.515$; $p<0.001)$, and a negative one between training level and time (Rho $=-0.589$; $p<0.001)$. Conclusions: More experienced and trained professionals had a better performance in a clinical analytical thinking test.
\end{abstract}

(Rev Med Chile 2011; 139: 455-461).

Key words: Clinical competence; Education, medical; Educational measurement.



4 razonamiento clínico ha sido identificado como un elemento fundamental en la práctica médica. Por este motivo los currículos de las escuelas de medicina han comenzado a adaptarse para dirigir la enseñanza hacia el aprendizaje del mismo ${ }^{1}$.

Si bien la evaluación es un componente esencial del proceso de aprendizaje, fortaleciéndolo y guiándolo ${ }^{2}$, presenta particular dificultad al dirigirse al razonamiento que lleva a la resolución de problemas clínicos. Este razonamiento involucra el desarrollo de hipótesis que serán utilizadas como guía para la recolección de los datos que llevarán a la confirmación o refutación de ellas ${ }^{3}$.

Existirían diferencias en ese proceso según el grado de entrenamiento: de esta manera, los médicos con mayor experiencia formarían sus hipótesis y plan diagnóstico/terapéutico rápidamente y con mayor precisión que aquellos con menos experiencia ${ }^{3}$.

Dada la dificultad en evaluar la adquisición de competencias en esta área, se han desarrollado herramientas específicas ${ }^{4}$. Varios formatos de examen han sido utilizados para evaluar el razonamiento clínico ${ }^{5}$, sin embargo, algunos de ellos han sido cuestionados, en algunos casos por no medir específicamente razonamiento médico y en otros, por fallas en la herramienta en sí. Esto último llevó a Groothoff y su equipo a desarrollar el MATCH (Measuring Analytical Thinking in Clinical Health Care $)^{7}$. Este método es novedoso, resuelve algunos de los puntos débiles de métodos de evaluación anteriores y ha sido empleado como evaluación de dicho proceso en forma confiable y eficiente, incluso comparando la capacidad re- 
solutiva de la prueba según niveles de formación de los examinados.

Groothoff desarrolló el MATCH basándose en el CIP (Comprehensive Integrative Puzzle) $)^{8}$, al considerar que otros métodos de evaluación del razonamiento clínico tienen cierto grado de incertidumbre, pues pueden inducir a que los alumnos adivinen las respuestas correctas. El MATCH corrige estas debilidades y mide razonamiento "hacia atrás" (backward reasoning) lo que sería, según el autor, comparable al razonamiento médico (es decir, partiendo de una hipótesis se obtienen datos clínicos que la validen $)^{7}$.

A pesar que la educación médica ha avanzado sustancialmente en los últimos 20 años explorando diferentes herramientas de evaluación, seguramente por lo reciente del desarrollo del MATCH, no hemos encontrado referencias sobre su desempeño en otros escenarios fuera del empleado en la descripción original. Dada la simplicidad de esta prueba y su potencial utilidad consideramos conveniente evaluar su desempeño.

El objetivo del presente trabajo fue valorar el desempeño de sujetos con diferente nivel de formación en pediatría, examinados por medio de MATCH y evaluar si existía asociación entre nivel de formación en pediatría y resultado en dicha prueba.

\section{Material y Método}

Estudio transversal que se llevó a cabo en los meses de marzo y abril de 2009 en un hospital pediátrico de la Ciudad de Buenos Aires, Argentina.

Se incluyeron cuatro grupos de sujetos con diferentes grados de formación en pediatría:

- Alumnos de la Facultad de Medicina de la Universidad de Buenos Aires que se encontraban cursando la última semana de la materia Pediatría en el hospital (duración total 8 semanas).

- Residentes de pediatría en el último mes de su primer año (R1), que se encontraban desarrollando sus tareas en el hospital.

- Residentes de pediatría en el último mes de su tercer año (R3), que se encontraban desarrollando sus tareas en el hospital.

- Médicos clínicos pediatras de planta del hospital, con al menos 5 años de finalizada su residencia, seleccionados al azar del listado de profesionales de la institución, hasta alcanzar el número de residentes de tercer año.
Se excluyeron aquellos que hubieran realizado con anterioridad un examen de tipo MATCH o que tuvieran, además, otra especialidad distinta de clínica pediátrica.

El MATCH administrado consistió en dos bloques de patologías (renales y respiratorias) que incluían 5 enfermedades cada uno y cinco ítems correspondientes a elementos necesarios para el diagnóstico y tratamiento de las mismas (anamnesis, clínica, laboratorio, imágenes, etc.). Cada ítem contenía siete opciones (5 correctas y 2 distractores). Para cada patología, los sujetos debían ubicar la opción más adecuada para cada uno de los ítems. Cada patología tenía sólo una opción correcta para cada ítem (Tabla 1). Por respuesta correcta se asignó un punto, por lo que el puntaje máximo de la prueba era 50 puntos. Para la construcción de la herramienta de evaluación sólo se incluyeron aspectos contenidos en el programa de la materia pediatría ${ }^{9}$. La herramienta de evaluación fue diseñada por instructores de residentes de pediatría y sus contenidos fueron supervisados por especialistas en cada uno de los temas contemplados y por especialistas en educación médica.

Para los fines del estudio se consideró variable de predicción el nivel en la formación en pediatría (cada uno de los grupos), y variables de resultado el puntaje obtenido en la prueba y el tiempo requerido para la resolución de la misma.

Consideraciones estadísticas: Se estimó un tamaño muestral mínimo de 16 sujetos para valorar diferencias de al menos 5 puntos en el resultado de la prueba y 5 minutos en el tiempo requerido para completarla, entre dos grupos. Se valoró puntaje obtenido y tiempo utilizado para resolver el examen, expresándolos en media y desvío estándar, mediana e intervalo intercuartílico, para cada grupo. Se evaluó la diferencia entre resultado de la prueba y tiempo requerido con el grado de entrenamiento por medio de la prueba de ANOVA o Kruskal-Wallis (de acuerdo ajuste a normalidad según prueba KolmogorovSmirnov). Las diferencias entre los grupos fueron evaluadas por medio de prueba $\mathrm{T}$ para muestras independientes o U de Mann-Whitney, según ajuste a normalidad. Además, se calculó la correlación (Spearman) entre el nivel de formación y cada una de las variables de resultado. Valor de significación $\mathrm{p}<0,05$. 
Tabla 1. Contenidos de la evaluación MATCH administrado a los participantes

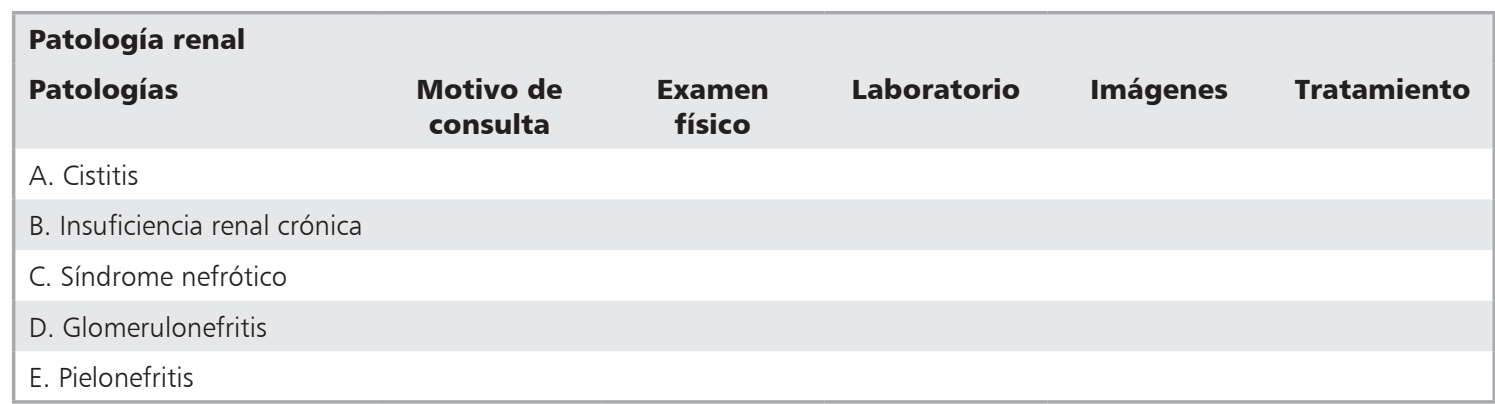

\section{Motivo de consulta:}

1. Paciente masculino de 10 años, con dolor cólico y orina oscura.

2. Paciente de 10 meses, masculino. Retraso de crecimiento, vómitos frecuentes y poliuria. Bebé dormilón.

3. Paciente femenino de 7 años, con orina oscura y antecedente de faringitis hace 2 semanas.

4. Paciente femenino de 10 años. Disuria de 24 horas de evolución. Sin antecedentes de episodios similares.

5. Paciente femenino de 5 años, con fiebre de 24 horas de evolución, dolor lumbar y disuria.

6. Paciente varón de 7 años. Edema en ojos de 48 horas de evolución más dolor abdominal.

7. Paciente femenino de 15 años con poliuria. Sin antecedentes patológicos.

\section{Examen físico:}

1. Edema en miembros inferiores, hipertensión arterial, púrpuras palpables en miembros inferiores.

2. Normal.

3. Edema bipalpebral en miembros inferiores, ascitis, hipotensión arterial.

4. Talla menor a -2 desvíos estándar, taquicardia, tensión arterial normal y palidez.

5. Temperatura $39,5^{\circ} \mathrm{C}$, puño-percusión positiva.

6. Edema en miembros inferiores, hipertensión arterial, adenopatías submaxilares.

7. Deshidratación, hipotensión, depresión del sensorio.

\section{Laboratorio:}

1. Proteinuria +++ , creatinina sérica normal.

2. Hematuria +++ , proteinuria ++ . Aumento de la creatinina plasmática.

3. Orina completa: $\delta 1,005$, proteínas + , sedimento: 12 leucocitos/campo. Aumento de creatinina y fosfatos plasmáticos.

4. Orina completa normal. Aumento de creatinina sérica.

5. Sedimento: 20 leucocitos/campo. Creatinina plasmática normal. Hiperleucocitosis.

6. Sedimento urinario: 20 leucocitos/campo, 7 eritrocitos/campo. Creatinina sérica normal.

7. Orina completa: $\delta 1,005$, proteínas +, sedimento: 12 leucocitos/campo. Aumento de hematocrito y fosfatos plasmáticos. Creatinina sérica normal.

\section{Imágenes:}

1. No es necesario.

2. RMN de abdomen.

3. No es necesario, pero eventualmente radiografía de tórax para evaluar estado de hidratación.

4. Ecografía y cistouretrografía.

5. Ecografía renal. Centellografía renal con DMSA.

6. Radiografía de tórax para evaluar estado de hidratación.

7. Ecografía renal.

\section{Tratamiento:}

1. Aporte de líquido, dieta hiposódica, aporte de vitamina D.

2. Trimetoprima-sulfametoxazol o nitrofurantoína o cefalexina.

3. Ciclofosfamida.

4. Glucocorticoides. Dieta hiposódica.

5. Antibioticoterapia de amplio espectro.

6. Rehidratación con reposición de electrolitos.

7. Restricción hídrica, dieta hiponatrémica. 
Tabla 1. Contenidos de la evaluación MATCH administrado a los participantes (continuación)

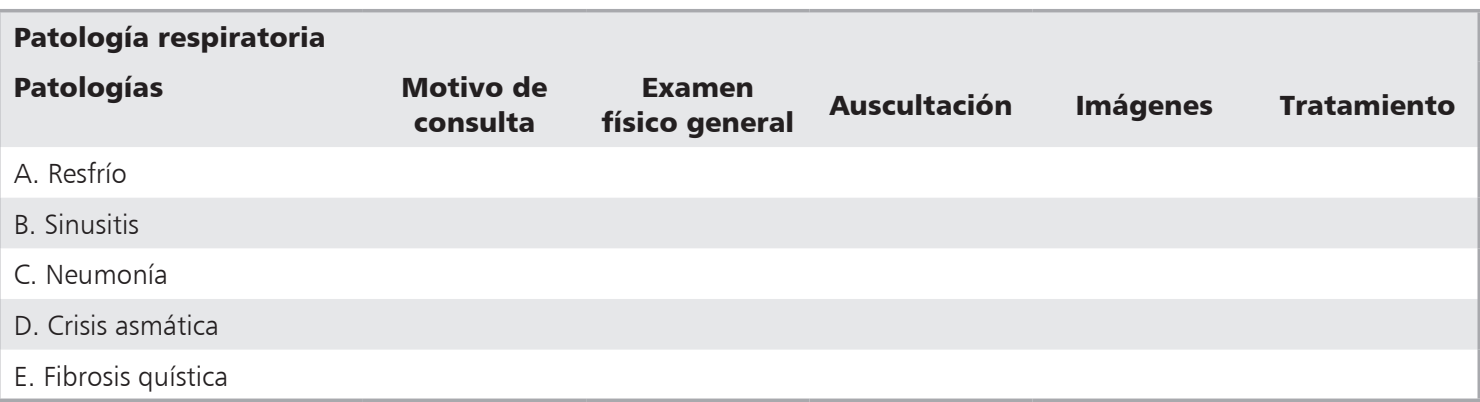

\section{Motivo de consulta:}

1. Paciente masculino de 6 meses con tos perruna y estridor.

2. Paciente femenina de 2 años de edad con fiebre, tos y decaimiento de 24 horas de evolución.

3. Paciente masculino de 14 años de edad con tos y rinorrea de 14 días de evolución.

4. Paciente de 2 años con rinorrea mucopurulenta unilateral.

5. Paciente masculino de 5 años con broncoespasmos a repetición.

6. Paciente femenina de 15 años de edad con disnea y tos de 8 horas de evolución

7. Paciente masculino de 8 meses de edad con rinorrea y fiebre de 48 horas de evolución.

\section{Examen físico general:}

1. Rinorrea mucopurulenta bilateral y tos.

2. Normal.

3. Tiraje intercostal y subcostal, taquipnea y taquicardia.

4. Rinorrea posterior. Temperatura $38^{\circ} \mathrm{C}$. Puntos maxilares dolorosos. Halitosis.

5. Regular estado general. Temperatura $39,5^{\circ} \mathrm{C}$. Tiraje intercostal. Taquicardia y taquipnea

6. Peso menor de -2 desvíos estándar, dedos en palillo de tambor, tórax en tonel. Taquicardia y taquipnea.

7. Taquipnea y taquicardia. Tiraje intercostal y supracalvicular. Babeo.

\section{Auscultación:}

1. Soplo anfórico en vértice derecho.

2. Buena entrada de aire bilateral sin ruidos agregados

3. Buena entrada de aire bilateral con roncus aislados.

4. Disminución de la entrada de aire con rales crepitantes en base derecha.

5. Disminución de la entrada de aire generalizada, sibilancias diseminadas y espiración prolongada.

6. Áreas de hipoventilación. Sibilancias y rales subcrepitantes dispersos. Roncus.

7. Disminución global de la entrada de aire, sin ruidos agregados.

\section{Imágenes:}

1. Ecografía pleural.

2. Radiografía de tórax descentrada de vértice.

3. Radiografía de tórax de frente.

4. No es necesario, eventualmente radiografía de tórax de frente.

5. Radiografía mento-naso-placa.

6. Radiografía de tórax de frente y posteriormente TAC.

7. No es necesario.

\section{Tratamiento:}

1. Fluidización de secreciones.

2. Amoxicilina $80 \mathrm{mg} / \mathrm{kg} /$ día por 10 días.

3. Oxígeno, salbutamol y glucocorticoides.

4. Claritromicina $15 \mathrm{mg} / \mathrm{kg} /$ día por 15 días.

5. Amoxicilina $1,5 \mathrm{~g} /$ día por 14 días.

6. Oxígeno, antibioticoterapia inhalada y sistémica.

7. Terapia antihistamínica más antitusiva. 


\section{Consideraciones éticas}

Se solicitó y obtuvo autorización de los comités de Docencia e Investigación y Bioética del hospital. El estudio fue registrado en el Consejo de Investigaciones en Salud del Gobierno de la Ciudad de Buenos Aires. La participación en el estudio fue voluntaria. Se informó por escrito a todos los participantes sobre los alcances del estudio y que la aceptación a participar de la prueba implicaba su consentimiento.

\section{Resultados}

Participaron del estudio 85 sujetos: 23 alumnos, 28 R1, 17 R3 y 17 médicos de planta. Parti- ciparon todos los alumnos que se encontraban cursando la materia en el hospital; no participaron 2 residentes de primer año, 1 residente de tercer año y 1 médico de planta por encontrarse ausentes el día de la prueba.

El puntaje promedio obtenido fue $37,4 \pm 6,0$ puntos, y el tiempo requerido para completar la prueba fue de 25,2 $\pm 8,5$ minutos (mediana: 22; rango intercuartílico: 18-32). Los puntajes seguían una distribución normal, no así los tiempos. Las diferencias entre grupos tanto para puntaje ( $p$ $<0,001)$ como para tiempo ( $\mathrm{p}<0,001)$ fueron estadísticamente significativas (Tabla 2) (Figuras 1 y 2 ).

Los médicos de planta fueron el grupo que alcanzó el mayor puntaje, pero sólo mostraron

Tabla 2. Puntaje obtenido y tiempo requerido para realizar la prueba MATCH, según grupos de estudio

\begin{tabular}{|lccc|}
\hline & Resultado (puntos*) & Tiempo (minutos**) \\
\hline Alumnos $(n=23)$ & $33,9 \pm 4,1$ & 35 & $(31-37)$ \\
Residentes de $1^{\circ}$ año $(n=28)$ & $37,0 \pm 7,4$ & 21 & $(18-27)$ \\
Residentes de $3^{\circ}$ año $(n=17)$ & $40,1 \pm 4,5$ & $18(13,5-20,5)$ \\
Médicos de planta $(n=17)$ & $41,8 \pm 3,1$ & 22 & $(17-26,5)$ \\
\hline
\end{tabular}

${ }^{*} X \pm \mathrm{DE} .{ }^{* *}$ Mediana (intervalo intercuartílico).

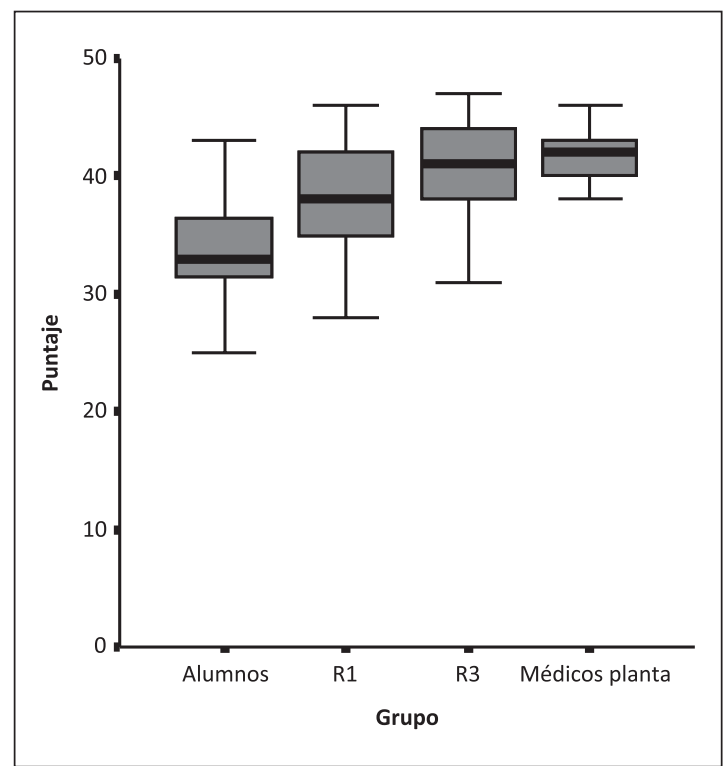

Figura 1. Puntaje en el MATCH según grado de formación en pediatría.

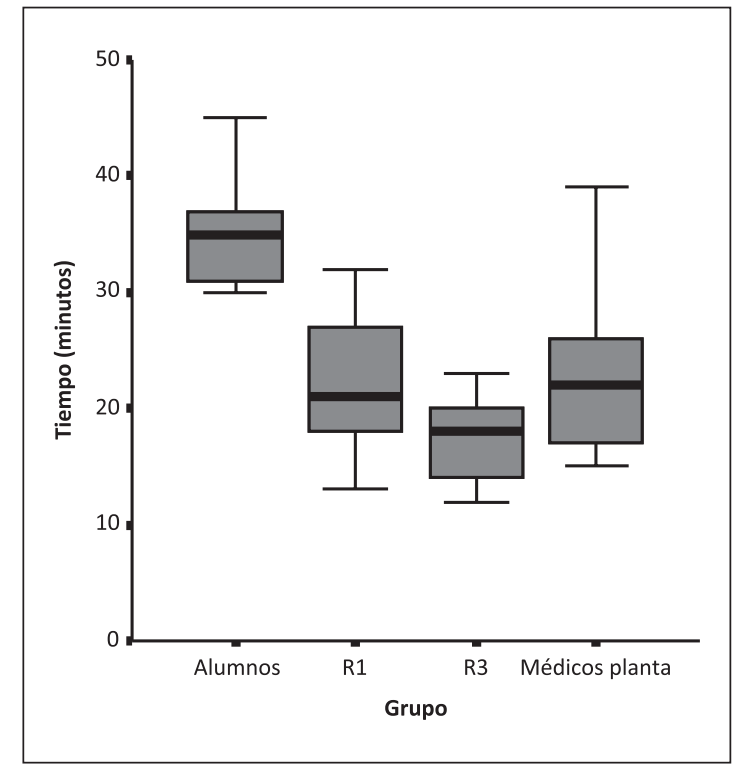

Figura 2. Tiempo requerido para completar el MATCH según grado de capacitación en pediatría. 
diferencias significativas con $\mathrm{R} 1(\mathrm{p}=0,01)$ y alumnos $(\mathrm{p}<0,001)$.

Los R3 fueron el grupo que empleo menos tiempo, mostrando diferencias significativas con cada uno de los otros grupos (alumnos: $\mathrm{p}<0,001$; $\mathrm{R} 1: \mathrm{p}=0,008$; médicos de planta: $\mathrm{p}=0,02$ ).

Se observó una correlación positiva entre grado de formación y el puntaje obtenido (Rho $=$ $0,515 ; \mathrm{p}<0,001)$. Por lo contrario, se observó una correlación negativa entre el grado de formación y el tiempo requerido para completar la prueba $($ Rho $=-0,589 ; \mathrm{p}<0,001)$.

\section{Discusión}

La prueba evaluada se mostró como una herramienta útil para valorar el razonamiento clínico. Encontramos que, en general, a mayor nivel de formación y experiencia se observó mayor puntaje en la prueba.

Esto coincide con lo referido por Drennan quien, al comparar graduados en una maestría de enfermería con alumnos que iniciaban la misma utilizando la WGCTA ${ }^{\text {UK }}$ (Watson-Glaser Critical Thinking Appraisal-versión UK), observó que los graduados obtuvieron mejores resultados que los alumnos $^{10}$. La WGCTA ${ }^{\mathrm{UK}}$, aunque desarrollada originalmente en la década de 1920, es aún una de las herramientas más utilizadas a nivel mundial para medir razonamiento crítico en educación ${ }^{11}$.

Por otro lado, resulta interesante analizar nuestros resultados considerando las características de los diferentes grupos participantes. De esta manera, se puede observar que los R3 y médicos de planta mostraron un desempeño similar. Esto puede deberse a que, en nuestro hospital, la formación de los R3 contempla específicamente coordinación de residentes más jóvenes, asumiendo la responsabilidad de las decisiones tomadas y, por lo tanto, ejercitando más su razonamiento clínico.

También encontramos que a mayor formación y experiencia disminuye el tiempo necesario para completar la prueba. Esto se verifica entre los tres primeros grupos evaluados. Sin embargo, los R3 constituyen el grupo que alcanzan el mejor tiempo, significativamente menor aún que los médicos de planta. Es posible que esto se deba a que, además de tratarse de patologías a las que ellos se enfrentan muy frecuentemente, estos residentes alcanzaron una razonable experiencia clínica, desarrollan una práctica intensiva y se ven sometidos a un constante entrenamiento en diferentes métodos de evaluación ${ }^{12}$. También podría deberse a que los médicos con mayor tiempo de ejercicio de la profesión pueden ser más metódicos y cautos a la hora de resolver situaciones clínicas, pudiendo esto influir en el tiempo final requerido para completar la evaluación.

Por otra parte, el análisis de los resultados (puntaje y tiempo) obtenidos por los alumnos de la carrera de medicina, parecería indicar que el conocimiento fundamentalmente teórico que ellos poseen no es suficiente para la resolución completa de problemas clínicos; aunque debe reconocerse que este estudio no incluyó una valoración de tal tipo de conocimiento entre los participantes. Sus resultados también podrían estar influenciados por una formación de grado que aún no está basada totalmente en la adquisición de competencias ni incluye sistemáticamente su evaluación ${ }^{13}$.

Nuestros resultados son consistentes con los obtenidos por Groothoff y col${ }^{7}$. Ellos aplicaron un MATCH a alumnos de medicina, residentes de pediatría y médicos pediatras, encontrando que el puntaje se incrementaba con el entrenamiento (obteniendo en promedio, sobre una escala de 100 puntos, 75,88 y 92, respectivamente). La progresión de los puntajes es similar a la obtenida por nosotros (sobre una escala de 50 puntos, $33,37 \mathrm{y}$ 41 , respectivamente).

Más aún, dadas las características de la enseñanza de pediatría en Holanda, ellos incluyeron alumnos de medicina de $4^{\circ}, 5^{\circ}$ y $6^{\circ}$ año, encontrando diferencias en su desempeño $(61,71$ y 75 puntos, respectivamente). Al igual que nuestro estudio, ellos también encontraron diferencias en el tiempo requerido para completar la prueba, donde los alumnos requirieron 49 minutos y los médicos 34 (en nuestro caso 35 y 22).

La competencia médica puede ser considerada como aquella que permite usar en forma habitual la comunicación, el conocimiento, las habilidades técnicas y el razonamiento clínico para resolver los problemas médicos que afectan a los individuos y a la salud pública. El proceso de adquisición de esta competencia, se desarrolla en el tiempo y depende en forma directa de la experiencia ${ }^{14}$.

Finalmente, es muy difícil definir cual es la relevancia de las diferencias encontradas por nosotros en cuanto a puntaje y velocidad en la prueba entre los diferentes grupos. Sin embargo, 
es razonable pensar que a mayor experiencia mejor razonamiento clínico.

Independientemente de las limitaciones que presenta todo sistema de evaluación, nuestro estudio sugiere que la experiencia que brinda el ejercicio de la profesión fortalece el proceso de aprendizaje continuo, logrando desarrollar el razonamiento médico exitosamente ${ }^{15}$.

En conclusión, los sujetos con mayor experiencia y formación profesional demostraron mayor capacidad de razonamiento clínico evaluado por una prueba específica (MATCH). Esta herramienta podría incluirse entre las estrategias para valorar razonamiento clínico en distintas instancias de la formación profesional.

Agradecimientos: Los autores agradecen al Dr. Carlos Castillo Durán la revisión crítica del manuscrito.

\section{Referencias}

1. Mamede S, Schmidt HG. Correlates of reflective practice in medicine. Adv Health Sci Educ Theory Pract 2005; 10: 327-37.

2. Bhakta B, Tennant A, Horton M, Lawton G, Andrich D. Using item response theory to explore the psychometric properties of extended matching questions examination in undergraduate medical education. BMC Med Educ 2005; 5: 9.

3. Elstein AS, Schwartz A. Clinical problem solving and diagnostic decision making: selective review of the cognitive literature. BMJ 2002; 324 (7339): 729-32.

4. Smith CS. A Developmental Approach to Evaluating Competence in Clinical Reasoning. J Vet Med Educ
2008; 35: 375-81.

5. Schuwirth LW, van der Vleuten CP. ABC of learning and teaching in medicine: Written assessment. BMJ 2003; 326 (7390): 643-5.

6. Kreiter CD, Bergus G. The validity of performance-based measures of clinical reasoning and alternative approaches. Med Educ 2009; 43: 320-5.

7. Groothoff JW, Frenkel J, Tytgat GA, Vreede WB, Bosman DK, Ten Cate OT. Growth of analytical thinking skills over time as measured with the MATCH test. Med Educ 2008; 42: 1037-43.

8. Ber R. The CIP (Comprehensive Integrative Puzzle) assessment method. Med Teach 2003; 25: 171-6.

9. Departamento de Pediatría. Contenidos de la materia agrupados por unidad temática. [Disponible en: http:// www.fmed.uba.ar/depto/pediatri/contenido.htm.] [Consulta: 2 de febrero de 2010].

10. Drennan J. Critical thinking as an outcome of a Master's degree in Nursing programme. J Adv Nurs 2010; 66: 422-31.

11. El Hassan K, Madhum G. Validating the Watson Glaser Critical Thinking Appraisal. Higher Education 2007; 54: 361-83.

12. Brinkman WB, Geraghty SR, Lanphear BP, Khoury JC, González del Rey JA, Dewitt TG, et al. Effect of Multisource Feedback on Resident Communication Skills and Professionalism. Arch Pediatr Adolesc Med 2007; 161: 44-9.

13. Reta-De Rosas AM, López MJ, Vargas AL, Montbrun MC. Evaluación de competencias médicas en un currículo de grado no diseñado por competencias. Educ Med 2008; 11: 207-18.

14. Epstein R. Assessment in Medical Education. N Engl J Med 2007; 356: 387-96.

15. Wass V, Van der Vleuten C, Shatzer J, Jones R. Assessment of clinical competence. Lancet 2001; (357): 945-9. 


\section{Referencias}

1. Martin W, Brynes S. Chilean Miners and Biomedical Research. A Modest Proposal. Am J Respir Crit Care Med 2010; 182: 1459-64.

2. Sobradillo P, Pozo F, Agustí A. Medicina P4: el futuro a la vuelta de la esquina. Arch Bronconeumol 2011; 47: 35-40.

Correspondencia a: Dr. Rafael Silva O.

Dos Norte 360, Talca. Fono: 71-412716 Fax: 71-209306

E-mail: rafaelsilvao@gmail.com 